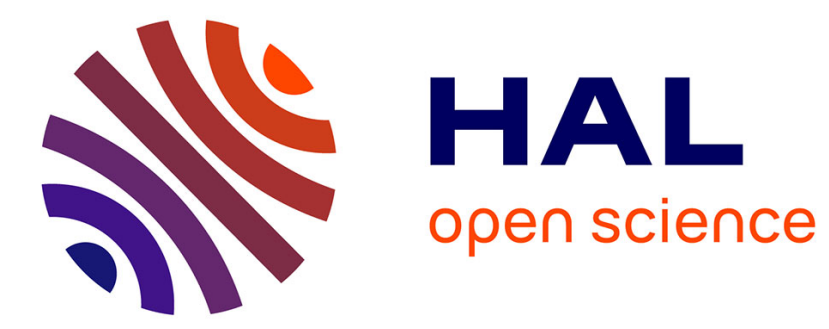

\title{
Sur l'électromètre à quadrants
}

M. Gouy

\section{- To cite this version:}

M. Gouy. Sur l'électromètre à quadrants. J. Phys. Theor. Appl., 1888, 7 (1), pp.97-109. 10.1051/jphystap:01888007009700 . jpa-00238930

\section{HAL Id: jpa-00238930 https://hal.science/jpa-00238930}

Submitted on 1 Jan 1888

HAL is a multi-disciplinary open access archive for the deposit and dissemination of scientific research documents, whether they are published or not. The documents may come from teaching and research institutions in France or abroad, or from public or private research centers.
L'archive ouverte pluridisciplinaire HAL, est destinée au dépôt et à la diffusion de documents scientifiques de niveau recherche, publiés ou non, émanant des établissements d'enseignement et de recherche français ou étrangers, des laboratoires publics ou privés. 


\section{SUR L'ÉLEGTROMÈTRE A QUADRANTS ;}

Par M. GOUY.

1. Objet du Mémoire. - D'après la théorie généralement admise, les forces électriques agissant sur l'aiguille de l'électromètre à quadrants se réduisent à un couple dont le moment est donné, à un facteur constant près, par l'expression

$$
\left(V_{1}-V_{2}\right)\left(V_{0}-\frac{V_{1}+V_{2}}{2}\right)
$$

en désignant par $V_{0}, V_{1}$ et $V_{2}$ les potentiels de l'aiguille et des deux paires de quadrants. Il en résulte que les petites déviations doivent être elles-mêmes proportionnelles à cette expression, ce que l'expérience confirme en général.

J'ai reconnu cependant que, dans un cas particulier assez important, la formule précédente se trouve entièrement en défaut; ce cas est celui de la charge symétrique, où les quadrants se trouvent maintenus à des potentiels assez élevés, égaux en valeur absolue, et de signes contraires, l'aiguille étant reliée à la source à étudier. On réalise ces condilions, comme on sait, en reliant les deux paires de quadrants aux deux pôles d'une pile d'un assez grand nombre d'éléments, dont le milieu est mis à la terre. D'après ce qui précède, la sensibilité devrait être proportionnelle au nombre d'éléments de la pile, et en raison inverse du couple de la suspension. On observe, au contraire, que la sensibilité est presque indépendante de ce couple, lorsqu'il est petit, et que, loin d'être proportionnelle au nombre des éléments, elle atteint un maximum pour un certain nombre d'éléments, et décroît rapidement quand ce nombre augmente.

Ces faits, remarqués en essayant de donner une grande sensibilité à l'électromètre, m'ont amené à faire une étude particulière de cet instrument. J'ai fait usage de deux électromètres à quadrants, du modèle de M. Mascart, construits par M. Carpentier, qui ne diffèrent que par la hauteur de la boîte formée par les quadrants, et d'un électromètre à secteurs plans. Comme pile de 
charge, j'ai employé d'abord une pile de $3 o o$ petits éléments Callaud, puis, afin de suivre plus loin les phénomènes, une pile de I ooo éléments au bioxyde de mercure valant $\mathrm{I}^{\text {volt }}, 40\left({ }^{1}\right)$.

2. Oscillations. - La charge symétrique produit une accélération manifeste des oscillations de l'aiguille, fait en contradiction avec la théorie généralement admise, et dont l'étude nous occupera d'abord.

Dans ces expériences, l'aiguille est reliée à la terre d'une manière permanente. On la fait osciller, d'abord sous l'action de la suspension seule, les quadrants étant mis à la terre, puis en les chargeant au moyen d'un nombre croissant d'éléments. On compte un certain nombre d'oscillations, on réduit l'amortissement le plus possible, ou on le supprime en remplaçant la suspension bifilaire par un fil métallique.

On trouve invariablement que la charge symétrique produit une accélération considérable. Ainsi, l'un des électromètres Mascart $\left({ }^{2}\right)$, à vide, a une période d'oscillation simple de $13^{\text {s }}$, avec uu fil de suspension en platine, de $\mathrm{o}^{\mathrm{mm}}, 02$ de diamètre; avec 300 éléments Callaud, la période devient $5^{\mathrm{s}}, 2$; elle se réduit à $1^{\mathrm{s}}, 34$ avec 1000 éléments au bioxyde de mercure.

Voici une série d'expériences faites avec cet électromètre et la pile de ı ooo éléments; $n$ désigne le nombre d'éléments employés, et $\theta$ la durée d'une oscillation simple, en secondes.

( ${ }^{1}$ ) Ces éléments ne diffèrent de l'élément étalon à bioxyde de mercure décrit ailleurs (Comptes rendus, 1887) que par leurs petites dimensions et par la suppression du mercure, l'électrode positive étant simplement formée d'un fil de platine.

$\left({ }^{2}\right)$ Modèle ordinaire, hauteur intéricure de la boite $: 9^{\mathrm{mm}}, 2$. 
TABLEIU I.

\begin{tabular}{|c|c|c|c|c|}
\hline$n$. & $\theta$. & $\frac{\mathrm{I}}{0^{2}}$. & $\frac{1}{\theta^{2}}-\frac{1}{\theta_{0}^{2}}$. & $\frac{\mathrm{r}}{n^{2}}\left(\frac{1}{\theta^{2}}--\frac{1}{\theta_{0}^{2}}\right)$ \\
\hline$o \ldots$ & 13,0 & 0,00592 & " & $\eta$ \\
\hline $100 \ldots$ & 9,4 & 0,0113 & 0,00538 & 0,000000538 \\
\hline $200 \ldots \ldots$ & $6, o$ & 0,0277 & 0,02178 & 0,000000544 \\
\hline $300 \ldots$ & 4,07 & 0,0603 & 0,05438 & 0,000000604 \\
\hline $400 \ldots$ & 3,3 & 0,0917 & 0,08578 & o, o0o0oo536 \\
\hline $500 \ldots$. & 2,62 & $o, 146$ & 0,14008 & o, 00000056o \\
\hline $600 \ldots$ & 2,21 & 0,205 & 0,19908 & 0,000000553 \\
\hline $700 \ldots$ & I, 9 f́ & 0,266 & 0,26008 & 0,00000053 I \\
\hline $800 \ldots$. & $\mathbf{I}, 69$ & 0,350 & 0,34408 & 0,000000537 \\
\hline $900 \ldots$ & I, 48 & 0,457 & $0,45 \mathrm{I} 08$ & 0,000000557 \\
\hline $1000 \ldots$. & 1,45 & 0,555 & 0,54908 & $o, 000000539$ \\
\hline
\end{tabular}

On constate que la durée d'oscillation ne dépend pas de l'amplitude, pour de petites oscillations. Le couple qui fait osciller l'aiguille est proportionnel à $\frac{\mathrm{I}}{\theta^{2}}$, dont les valeurs sont données dans la troisième colonne; on voit que ce couple varie de i à ıoo dans les limites des expériences. Il faut donc qu'il existe, en même temps que le couple directeur, dû à la suspension, un autre couple directeur, dû aux forces électriques, qui tend aussi à ramener l'aiguille au zéro, et qui agit proportionnellement à l'angle d'écart; nous l'appellerons couple directeur électrique. Le moment de ce couple est proportionnel à $\frac{\mathrm{I}}{\theta^{2}}-\frac{\mathrm{I}}{\theta_{0}^{2}}$, en désignant par $\theta_{0}$ la durée de l'oscillation à vide; sa valeur est donnée par la quatrième colonne.

Le couple directeur électrique est indépendant de la suspension, comme on pouvait le prévoir. Ainsi le même électromètre, avec un fil plus gros $\left(0^{\mathrm{mm}}, 05\right)$, a une période, à vide, de $3^{\mathrm{s}}, \mathrm{I}$, et, avec ıooo éléments, de $\mathrm{I}^{\mathrm{s}}, 25$. La valeur du couple directeur électrique, d'après cela, est de 0,537 , en employant les unités précédentes.

Avec une suspension formée d'un seul fil de cocon, le couple de la suspension est tout à fait négligeable, et le couple électrique agit seul; la période est de $\mathbf{1}^{\mathrm{s}}, 37$ avec $\mathbf{~ o ́ o o ~ e ́ l e ́ m e n t s , ~ e t ~ l a ~ v a l e u r ~}$ du couple de o, $\tilde{5}_{2}$. L'appareil ainsi disposé fournit une démonstration très simple de l'existence du phénomène que nous étudions. 
Le couple directeur électrique est proportionnel au carré du nombre d'éléments de la pile de charge. - La cinquième colonne du Tableau précédent donne le quotient du moment de ce couple par $n^{2}$, et cette quantité est constante, au degré d'approximation que comporte la mesure de $\theta$. Cette loi s'est trouvée confirmée par toutes les expériences, et nous verrons plus loin qu'elle est d'accord avec la théorie.

Il nous reste à examiner comment varie ce couple avec la distance de l'aiguille aux quadrants. Avec les électromètres ordinaires, l'aiguille étant au milieu de la boîte formée par les quadrants, cette distance est la moitié de la hauteur de la boîte. Dans l'électromètre auquel se rapportent les résultats donnés plus haut. cette hauteur est de $9^{\mathrm{mm}}, 2$; elle est de $4^{\mathrm{mm}}, 8$ dans un autre électromètre, d'ailleurs identique. Les deux appareils, avec la même aiguille suspendue par un fil de cocon, donnent sensiblement la même période d'oscillation $\left(\mathrm{I}^{\mathrm{s}}, 37\right.$ et $\left.\mathbf{1}^{\mathrm{s}}, 38\right)$ avec rooo éléments.

L'électromètre à secteurs plans (1) permet de faire varier la distance dans des limites plus étendues :

Durée de l'oscillation, à vide................... $8{ }_{8,7}^{8}$

\begin{tabular}{|c|c|c|c|c|c|}
\hline ") & ” & avec 1000 & éléments, & distance & $13^{\mathrm{mm}}$ \\
\hline ") & " & $"$ & 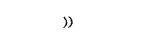 & " & $9^{\mathrm{mm}}$ \\
\hline " & 》) & $"$ & 》) & " & $5^{\mathrm{mm}}$ \\
\hline 1) & ” & ” & ” & " & $3^{\mathrm{mm}}$ \\
\hline » & 》) & ” & ” & » & $2^{\mathrm{mm}}$ \\
\hline
\end{tabular}

On peut conclure de ces diverses expériences que le couple directeur électrique varie en sens inverse de la distance de l'aiguille aux secteurs, mais que cette variation est peu sensible quand la distance est petite.

En résumé, la charge symétrique produit un couple, proportionnel au carré de la différence de potentiel des quadrants, qui tend à ramener l'aiguille dans une position symétrique par rapport aux quadrants $\left({ }^{2}\right)$. L'existence de ce couple, que ne faisait

(1) L'aiguille de ces électromètres est assez lourde, en forme de 8; celles des deux autres sont légères, de la forme figurée dans l'Ouvrage de MM. Mascart et Joubert. Il ne paraît pas que la forme de l'aiguille joue un rôle très important dans le phénomène, mais cette question mériterait une étude particulière.

( ${ }^{2}$ ) En chargeant l'aiguille à de très hauts potentiels, le quadrant étant relié à 
pas prévoir la théorie usuelle, va nous rendre compte de la marche de l'instrument, employé comme électromètre.

3. Déviations. - Avec la charge symétrique, la sensibilité de l'électromètre croît d'abord proportionnellement au nombre d'éléments de la pile de charge, puis, de moins en moins vite, passe par un maximum pour un certain nombre d'éléments, et décroît ensuite. Ainsi, avec l'électromètre auquel se rapporte le Tableau I, on a une déviation de $46^{\mathrm{mm}}$ pour $\mathrm{i}$ daniell (l'échelle étant à $2^{\mathrm{m}}$ ), avec une pile de charge de 30 éléments Callaud; avec 6o éléments, la déviation est de $8 \mathrm{o}^{\mathrm{mm}}$; le maximum est atteint vers $\mathrm{s} 5 \mathrm{o}$ éléments, il est de $106^{\mathrm{mm}}$; puis, avec les 3 oo éléments, la déviation se réduit à $8 \mathrm{o}^{\mathrm{mm}}$.

Ces variations sont encore plus étendues avec la pile de ı ooo éléments. Le Tableau suivant donne les déviations, pour I daniell, avec ce même électromètre muni de trois suspensions différentes :

I, un seul fil de cocon.

II, fil de platine de $\mathrm{o}^{\mathrm{mm}}$, o2.

III, fil de platine de $\mathrm{o}^{\mathrm{mm}}, 04$.

$n$ désigne le nombre d'éléments de la pile de charge.

TABleau II.

\begin{tabular}{|c|c|c|c|}
\hline$n$. & I. & II. & III. \\
\hline $20 \ldots \ldots \ldots \ldots$ & " & 42,5 & " \\
\hline $60 \ldots \ldots \ldots$ & ") & 97,5 & ") \\
\hline $100 \ldots \ldots \ldots$ & $23 \mathrm{I}, 7$ & I I I, 5 & 12,3 \\
\hline $140 \ldots \ldots \ldots$ & 1) & 107,5 & " \\
\hline $200 \ldots \ldots \ldots \ldots$ & I I 6,0 & $9^{2}, 0$ & $2 \mathbf{I}, \mathrm{O}$ \\
\hline $300 \ldots \ldots \ldots$ & 77,7 & 70,0 & 25,8 \\
\hline $400 \ldots \ldots \ldots$ & 58,0 & 54,2 & $27, \mathrm{o}$ \\
\hline $500 \ldots \ldots \ldots$ & ") & 45,5 & 26,5 \\
\hline $600 \ldots \ldots \ldots$ & $39, \mathbf{o}$ & 38,0 & 25,8 \\
\hline $700 \ldots \ldots \ldots \ldots$ & $"$ & 33,0 & 24,3 \\
\hline $800 \ldots \ldots \ldots \ldots$ & 29,2 & 28,6 & 22,9 \\
\hline $900 \ldots \ldots \ldots$ & $"$ & $25, f$ & 21,3 \\
\hline $1000 \ldots \ldots \ldots$ & 23,3 & 23,0 & 19,7 \\
\hline
\end{tabular}

la terre, la période d'oscillation est sensiblement la même qu'à vide, quand l'appareil est bien réglé. En général, il y a une différence assez appréciable, soit en plus, soit en moins. Ainsi, la période à vide étant de trcize secondes, elle a varié, avec rooo éléments, de onze à quinze secondes, suivant le réglage de l'appareil; avec une centaine d'éléments, ces différences sont insensibles. 
Ces résultats sont représentés dans la figure ci-dessous; $n$ est porté en abscisse et la déviation en ordonnée. Les droites ponctuées, tangentes aux courbes II et III à l'origine, représentent la déviation que donne I daniell lorsqu'on charge, non plus les quadrants, mais l'aiguille, avec la même pile de charge.

Avec la suspension I, dont le couple est nul, la déviation est en raison inverse du nombre $n$ des éléments de la pile de charge, et la courbe correspondante est une hyperbole équilatère.

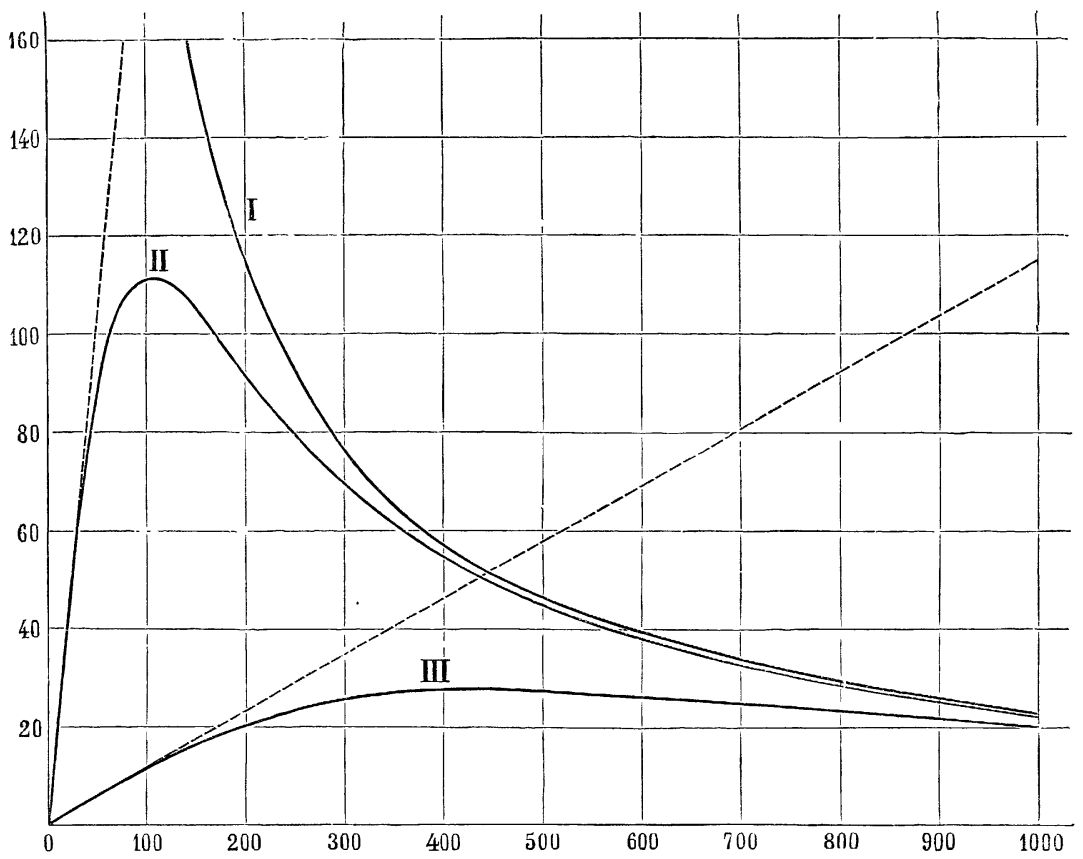

Ce résultat nous montre que le couple qui tend à dévier l'aiguille est proportionnel à $n$, conformément à la théorie usuelle de l'électromètre. En effet, ce couple étant équilibré par le couple directeur électrique, qui agit seul ici pour ramener l'aiguille au zéro, et qui est exprimé par $\mathrm{A} n^{2}$, A étant une constante, la déviation sera proportionnelle à $\frac{n}{\mathrm{\Lambda} n^{2}}=\frac{\mathrm{I}}{\mathrm{A} n}$.

Avec les suspensions II et III, les effets sont plus complexes. Le couple qui tend à dévier l'aiguille est évidemment le même 
que précédemment, mais le couple directeur est ici donné par l'expression A $n^{2}+$ B. B étant le moment du couple de la suspension. La déviation sera donc proportionnelle à $\frac{n}{\mathrm{~A} n^{2}+\mathrm{B}}$.

Il en résulte que la déviation est proportionnelle à $n$, quand $n$ est petit; qu'elle passe par un maximum pour $n=\sqrt{\frac{\overline{\mathrm{B}}}{\mathrm{A}}}\left({ }^{1}\right)$, et qu'elle tend vers la limite $\frac{\mathrm{I}}{\mathrm{A} n}$, quand $n$ s'accroît; c'est la valeur qu'elle possédait avec la suspension I. Aussi l'on voit que les courbes II et III sont asymptotes à l'hyperbole I.

On voit aussi que, A étant indépendant de la suspension, la valeur de $n$ qui produit le maximum de sensibilité est proportionnelle à $\sqrt{\mathbf{B}}$. Ainsi, avec une pile donnée, le maximum ne sera observable que lorsque le couple de la suspension sera petit, c'està-dire quand l'instrument sera réglé pour avoir une grande sensibilité; dans le cas contraire, par exemple avec l'électromètre disposé pour l'étude de l'électricité atmosphérique, la portion initiale de la courbe sera seule observable, et l'instrument obéira sensiblement à la formule usuelle.

En résumé, avec la charge symétrique, le couple qui tend à dévier l'aiguille est proportionnel à la différence de potentiel des quadrants, comme l'indiquait la théorie; mais le couple directeur électrique, dont elle ne tenait pas compte, modifie complètement les phénomènes, lorsque le couple de la suspension n'est pas assez grand pour le rendre négligeable.

3. Remarque sur l'usage de l'électromètre. - Il résulte de ce qui précède que la charge symétrique ne permet pas de donner à l'électromètre une très grande sensibilité, puisque cela ne pourrait être obtenu qu'avec un petit nombre d'éléments de la pile de charge, et, par suite, avec des forces trop petiles pour que l'appareil fût d'un bon usage. Mais la sensibilité qu'on peut obtenir est bien suffisante pour la plupart des expériences $\left({ }^{2}\right)$, et nous

(1) On voit aisément que ce maximum est égal à la moitié de la déviation qui existerait si le couple directement électrique n'agissait pas, avec le mêmo nombre d'éléments.

$\left({ }^{2}\right)$ Cette sensibilité est environ deux fois plus grande avec l'électromètre dont la boite a une hauteur de $4^{\mathrm{mm}}, 8$. 
allons voir que la charge symétrique présente certains avantages dans la pratique.

En premier lieu, on peut rendre la sensibilité presque indépendante des variations de la pile de charge en se plaçant au voisinage du maximum; avec une suspension formée d'un fil métallique, la sensibilité devient ainsi à peu près invariable.

Mais on peut en tirer un meilleur parti en suspendant l'aiguille par un fil métallique très fin $\left(\mathrm{o}^{\mathrm{mm}}, 02\right)$, et rendant le couple directeur électrique assez notable, de telle sorte qu'il agisse presque seul pour diriger l'aiguille. L'expérience montre que ce couple directeur n'est pas sujet aux mêmes irrégularités que les suspensions, bifilaire ou unifilaire, irrégularités qui produisent en majeure partie les variations du zéro de l'instrument et limitent la précision des mesures.

Toutefois, pour obtenir la fixité du zéro, il est indispensable que les deux moitiés de la pile soient égales, ou présentent une différence très petite et constante, ce qu'il est très difficile de réaliser. En effet, avec une pile de 200 éléments, par exemple, il suffit que l'une des moitiés de la pile varie seule de $o^{\text {volt }}, 02$, c'està-dire de $o^{\text {volt }}, 0002$ par élément, pour que le zéro se déplace d'une quantité correspondant à $\mathrm{o}^{\text {volt }}, \mathrm{O} \mathrm{I}$; or de pareilles variations résultent presque inévitablement des trépidations ou autres causes perturbatrices qui agissent inégalement sur les deux moitiés de la pile.

On peut lever cette difficulté d'une manière complète en reliant les quadrants aux deux extrémités d'une grande résistance métallique, traversée par un courant, et dont le milieu est relié à la terre. La pile qui fournit le courant doit être placée sur un pied isolant. Je fais usage pour cela, depuis plusieurs années, d'une pile de 3 oo éléments Callaud, de $30^{\mathrm{cm}}$ de hauteur sur $2^{\mathrm{cm}}$ de diamètre, et d'une boîte de résistance de I mégohm. Le courant étant très faible, il n'y a pas d'échauffement sensible des bobines, et les potentiels aux deux extrémités de la boîte restent exactement égaux en valeur absolue.

Ce dispositif, à défaut de simplicité, présente l'avantage de donner au zéro de l'instrument une fixité qu'on ne pourrait guère obtenir, par d'autres moyens. Les déplacements, dans le cours d'une séance d'observations, dépassant rarement quelques dixièmes 
de millimètre, on peut aisément mesurer $o^{\text {volt }}$, oo I. On peut employer avec avantage de l'huile au lieu d'acide sulfurique, pour amortir les oscillations de l'aiguille.

1. Théorie. - Les phénomènes décrits précédemment n'étant pas d'accord avec la théorie usuelle, nous devons penser que les conditions que suppose celte théorie ne sont pas suftisamment réalisées, et nous allons essayer de traiter la question à un point de vue plus général, en nous appuyant seulement sur des considérations de symétrie. Nous admettrons que la forme de l'instrument est telle que tout est symétrique par rapport à un certain plan vertical pour une position déterminée de l'aiguille, position que nous prendrons pour zéro, et qui, lorsque l'instrument est bien réglé, correspond à l'équilibre de la suspension.

Supposons d'abord l'aiguille maintenue au zéro par une force extérieure quelconque. Soient $\mathbf{M}_{0}, \mathbf{M}_{1}, \mathbf{M}_{2}$ les charges de l'aiguille et des deux paires de quadrants, $\mathrm{V}_{0}, \mathrm{~V}_{1}, \mathrm{~V}_{2}$ leurs potentiels, $\mathrm{C}_{0}$, $\mathrm{C}_{1}, \mathrm{C}_{2}$ leurs capacités, $i_{1}, i_{2}$ les coefficients d'induction entre l'aiguille et les deux paires de quadrants, $i_{q}$ le même coefficient entre les deux paires de quadrants ( 1 ).

D'après un théorème connu, on a

$$
\left\{\begin{array}{l}
\mathrm{M}_{0}=\mathrm{G}_{0} \mathrm{~V}_{0}-i_{1} \mathrm{~V}_{1}+i_{2} \mathrm{~V}_{2}, \\
\mathrm{I}_{1}=i_{1} \mathrm{~V}_{0}-\mathrm{C}_{1} \mathrm{~V}_{1}-i_{q} \mathrm{~V}_{2}, \\
\mathrm{M}_{2}=i_{2} \mathrm{~V}_{0}-i_{q} \mathrm{~V}_{1}-\mathrm{C}_{2} \mathrm{~V}_{2},
\end{array}\right.
$$

et l'éncrgie électrique W du système sera

$$
W=\frac{I}{2}\left(M_{0} V_{0}-M_{1} V_{1}+M_{2} V_{2}\right) .
$$

Faisons maintenant tourner l'aiguille a'un angle très petit ò, les potentiels restant constants. Le travail $\mathrm{T}$ produit par les forces électriques est donné par l'équation

$\mathrm{T}=\Delta \mathrm{W}=\frac{\mathrm{r}}{2}\left(\Delta \mathrm{C}_{0} \mathrm{~V}_{0}^{2}+\Delta \mathrm{C}_{1} \mathrm{~V}_{1}^{2}+\Delta \mathrm{C}_{2} \mathrm{~V}_{2}^{2}\right)+\Delta i_{1} \mathrm{~V}_{0} \mathrm{~V}_{1}+\Delta i_{2} \mathrm{~V}_{0} \mathrm{~V}_{2}+\Delta i_{q} \mathrm{~V}_{1} \mathrm{~V}_{2}$

( ${ }^{1}$ ) Les autres conducteurs du système, tels que la cage de l'instrument, sont supposés en communication avec le sol, et il est inutile d'en tenir compte dans les formules qui suivent. 
en désignant par $\Delta \mathrm{C}_{0}, \Delta \mathrm{C}_{1}, \ldots, \Delta i_{q}$ les variations des coefficients $\mathrm{C}_{0}, \mathrm{C}_{1}, \ldots, i_{q}$ produites par la rotation $\delta$. Ces variations sont assujetties à certaines conditions, en raison de la symétrie du système. $\Delta \mathrm{C}_{0}$ ne dépend pas du signe de $\delta$; on pourra donc poser, en négligeant les puissances de $\delta$ supérieures à la deuxième,

on aura de même

$$
\Delta \mathrm{C}_{0}=x \hat{\delta}^{2}
$$

$$
\Delta i_{q}=\beta \delta^{2} .
$$

Quant à $\Delta \mathrm{C}_{1}$ et $\Delta \mathrm{C}_{2}$, ils échangent leurs valeurs quand ò change de signe; on aura donc

et de même

$$
\begin{array}{lr}
\Delta \mathrm{G}_{1}= & \gamma \hat{0}+\lambda \delta^{2}, \\
\Delta \mathrm{C}_{2}= & -\gamma \hat{\partial}-\lambda \hat{o}^{2},
\end{array}
$$

$$
\begin{aligned}
& \Delta i_{1}=\mu \hat{o}+v \hat{o}^{2}, \\
& \Delta i_{2}=-\mu \hat{o}-v \hat{o}^{2},
\end{aligned}
$$

$\alpha, \beta, \gamma, \lambda, \mu$ et $\nu$ étant des coefficients caractéristiques de l'appareil.

II vient donc

$$
\left\{\begin{aligned}
T= & {\left[\frac{\mathrm{I}}{2} \gamma\left(\mathrm{V}_{1}^{2}-\mathrm{V}_{2}^{2}\right)+\mu \mathrm{V}_{0}\left(\mathrm{~V}_{1}-\mathrm{V}_{2}\right)\right] \delta } \\
& +\left[\frac{\mathrm{I}}{2} x \mathrm{~V}_{0}^{2}+\frac{\mathrm{I}}{2} \lambda\left(\mathrm{V}_{1}^{2}+\mathrm{V}_{2}^{2}\right)-\nu \mathrm{V}_{0}\left(\mathrm{~V}_{1}+\mathrm{V}_{2}\right)+\beta \mathrm{V}_{1} \mathrm{~V}_{2}\right] \delta^{2} .
\end{aligned}\right.
$$

Si l'on pose

$$
\left\{\begin{array}{l}
\mathrm{C}=\frac{\mathrm{I}}{2} \gamma\left(\mathrm{V}_{1}^{2}-\mathrm{V}_{2}^{2}\right)-\mu_{0} \mathrm{~V}_{0}\left(\mathrm{~V}_{1}-\mathrm{V}_{2}\right), \\
\mathrm{C}^{\prime}=\alpha \mathrm{V}_{0}^{2}-\lambda\left(\mathrm{V}_{1}^{2}+\mathrm{V}_{2}^{2}\right)-2 \nu \mathrm{V}_{0}\left(\mathrm{~V}_{1}-\mathrm{V}_{2}\right)+2,3 \mathrm{~V}_{1} \mathrm{~V}_{2} .
\end{array}\right.
$$

l'équation (3) peut s'écrire

$$
\mathrm{T}=\int_{0}^{\grave{\delta}}\left(\mathrm{C}+\mathrm{C}^{\prime} \grave{\delta}\right) d \hat{\mathrm{c}}
$$

Cette équation nous montre que les forces électriques appliquées à l'aiguille peuvent être réduites à deux couples agissant simultanément :

I $^{\circ}$ Un couple dont le moment $\mathrm{C}$ est indépendant de ò; il tend à 
dévier l'aiguille dans le sens des $\delta$ positifs, si $\mathrm{G}>\mathbf{o}$; dans le sens contraire si $\mathrm{G}<\mathrm{o}$.

$2^{\circ}$ Un couple dont le moment $\mathrm{C}^{\prime} \delta$ change de signe avec $\delta$ et s'annule pour $\delta=0$; c'est le couple directeur électrique dont nous avons reconnu l'existence expérimentalement. Il tend à ramener l'aiguille au zéro, de quelque côté qu'elle s'en écarte, si l'on a $\mathrm{C}^{\prime}<\mathrm{o}$, et à l'écarter toujours du zéro, si l'on a $\mathrm{C}^{\prime}>\mathbf{o}$.

Si l'aiguille avait de très grandes dimensions, comme le suppose la théorie usuelle, il est aisé de voir que l'on aurait

$$
\alpha=\beta=\lambda=\nu=o, \quad \text { et } \quad \mu=-\gamma .
$$

Il viendrait donc, dans ce cas,

et

$$
\mathrm{C}^{\prime}=\mathrm{o}
$$

$$
\mathrm{G}=\gamma\left[\frac{\mathrm{I}}{2}\left(\mathrm{~V}_{1}^{2}-\mathrm{V}_{2}^{2}\right)-\mathrm{V}_{0}\left(\mathrm{~V}_{1}-\mathrm{V}_{2}\right)\right]=-\gamma\left(\mathrm{V}_{1}-\mathrm{V}_{2}\right)\left(\mathrm{V}_{0}-\frac{\mathrm{V}_{1}+\mathrm{V}_{2}}{2}\right)
$$

ce qui est l'expression usuelle. C'est donc la limitation de l'aiguille qui produit dans le fonctionnement de l'appareil les effets que nous étudions.

Examinons maintenant quelques cas particuliers, en vue de connaître le rôle des divers coefficients qui entrent dans les formules (4).

Si l'on fait osciller l'aiguille en la chargeant et mettant les quadrants à la terre, il vient $\mathrm{C}=0, \mathrm{C}^{\prime}=\alpha \mathrm{V}_{2}^{0}$. L'aiguille oscille sous l'action du couple $\mathrm{C}^{\prime}$ et de la suspension. L'expérience montre que $\mathrm{C}^{\prime}$ est peu différent de zéro (1). On pouvait le prévoir en remarquant que la capacité $\mathrm{C}_{0}$ de l'aiguille est indépendante de $\delta$, si l'instrument est bien construit et réglé; c'est-à-dire si la boîte formée par les quadrants est de révolution autour de l'axe de rotation de l'aiguille, les fentes qui séparent les quadrants étant trop petites pour avoir un effet sensible; on doit donc avoir sensiblement

$$
\alpha=0 .
$$

(') Page roo, en note.

I. de Phys., $2^{\mathbf{B}}$ sèrie, t. VII. (Mars 1888. .) 
Il en serait évidemment de même si l'on faisait $V_{0}=0$, $\mathrm{V}_{1}=\mathrm{V}_{2}$; on doit donc avoir sensiblement, sous les mêmes conditions,

$$
\lambda=-\beta .
$$

Il en serait encore de même pour $\mathrm{V}_{0}=\mathrm{V}_{1}=\mathrm{V}_{2}$; on a donc sensiblement

$$
\nu=0 \text {. }
$$

Remarquons enfin que l'équilibre de l'aiguille ne dépend que des différences des potentiels $V_{0}, V_{1}$ et $V_{2}$, et non de leurs valeurs absolues, pourvu que l'aiguille soit efficacement protégée par la boîte formée par les quadrants ( 1 ); on doit donc avoir $\mathrm{G}=$ o pour $\mathrm{V}_{0}=\frac{\mathrm{V}_{1}+\mathrm{V}_{2}}{2}$, et par suite

$$
\gamma=-\mu
$$

En tenant compte de ces remarques, on obtient les expressions très approchées

$$
\left\{\begin{array}{l}
\mathrm{C}=-\gamma\left(\mathrm{V}_{1}-\mathrm{V}_{2}\right)\left(\mathrm{V}_{0}-\frac{\mathrm{V}_{1}+\mathrm{V}_{2}}{2}\right) \\
\mathrm{C}^{\prime}=\lambda\left(\mathrm{V}_{1}-\mathrm{V}_{2}\right)^{2} .
\end{array}\right.
$$

L'expression de C est celle que donne la théorie usuelle.

Oscillations avec la charge symétrique. - L'aiguille étant mise à la terre, on a

et, par suite,

$$
\mathrm{V}_{0}=\mathrm{o}, \quad \mathrm{V}_{1}=-\mathrm{V}_{2}
$$

$$
\mathrm{G}=\mathrm{o} \quad \text { et } \quad \mathrm{C}^{\prime}=2 \mathrm{~V}_{1}^{2}(\lambda-\beta),
$$

ou sensiblement

$$
\mathrm{C}^{\prime}=4 \lambda \mathrm{V}_{\mathbf{1}}^{2}
$$

Le couple directeur électrique $\mathrm{C}^{\prime}$ est proportionnel au carré de la différence de potentiel des quadrants, comme l'a montré l'expérience. Bien que $\lambda$ soit sans doute fort petit en valeur absolue, ce couple peut devenir très notable si $\mathrm{V}_{1}^{2}$ est très grand.

( ${ }^{1}$ Ou bien assez éloignée de la cage de l'instrument pour que celle-ci n'ait pas d'effet appréciable. 
Déviation. - En désignant, comme précédemment, par B le couple de la suspension, le moment du couple qui tend à ramener l'aiguille au zéro est $\left(B-C^{\prime}\right) \delta(1)$; le couple $C$ tend à l'en écarter ; par suite, l'équation d'équilibre sera

$$
\delta=\frac{\mathrm{G}}{\mathrm{B}-\mathrm{G}^{\prime}} .
$$

Avec la charge symétrique, cette cxpression devient, en négligeant le terme $\alpha V_{0}^{2}$, qui est ici tout à fait insensible, $V_{0}$ n'étant pas très grand,

ou sensiblement

$$
\delta=\frac{2 \mu V_{0} V_{1}}{B-2(\lambda-\beta) V_{1}^{2}}
$$

$$
\delta=-\frac{\lambda V_{0} V_{1}}{B-2 \lambda V_{1}^{2}}
$$

expression de même forme que celle que nous avait donnée l'expérience.

Avec la charge de l'aiguille, l'équation (6) ne peut pas être simplifiée dans le cas général. Mais, si les potentiels des quadrants ne sont pas très différents, $\mathrm{C}^{\prime}$ sera très petit, et, dans l'hypothèse contraire, $B$ est nécessairement très grand, de telle sorte que la formule usuelle sera applicable.

En reliant l'aiguille à un des quadrants, et mettant l'autre à la terre, suivant la méthode de M. Joubert, on aura

et par suite

$$
\mathrm{V}_{0}=\mathrm{V}_{1}, \quad \mathrm{~V}_{2}=\mathrm{o},
$$

$$
\delta=-\frac{\gamma \mathrm{V}_{0}^{2}}{2\left(\mathrm{~B}_{2}-\lambda \mathrm{V}_{0}^{2}\right)}
$$

il est facile de voir que le terme de $\lambda V_{0}^{2}$ sera insensible, pour les mêmes raisons.

En résumé, avec un instrument bien construit, deux coefficients seulement, $\gamma$ et $\lambda$, sont nécessaires à connaître pour calculer les effets de l'appareil. Le premier est donné par la théorie usuelle; le second, qui joue un rôle essentiel dans la charge symétrique, paraît au contraire fort difficile à calculer, mais on peut aisément les déterminer tous deux par l'expérience.

(') On se souvient que $\mathrm{G}^{\prime}<$ o quand le couple directeur électrique tend à ramener l'aiguille au zéro. 